\title{
Solar SW radiative transfer in bubbled ice: spectral considerations, subsurface enhancement, and inclusions - Supplementary Material
}

\author{
Andrew R.D. Smedley ${ }^{1,2}$, Geoffrey W. Evatt ${ }^{2}$, Amy Mallinson ${ }^{2}$, and Eleanor Harvey ${ }^{2}$ \\ ${ }^{1}$ School of Earth and Environmental Sciences, University of Manchester, Manchester, M13 9PL, UK \\ $5 \quad{ }^{2}$ School of Mathematics, University of Manchester, Manchester, M13 9PL, UK
}

Correspondence to: Andrew R.D. Smedley (andrew.smedley@manchester.ac.uk)

\section{S1 Solar zenith angle dependence}

Here we investigate the influence of the incident solar zenith angle. For simplicity, the results presented in the main text have relied on the assumption that the incoming solar flux arrives from a diffuse hemispherical sky, with no direct component.

10 However the angular dependence of Fresnel reflection coefficients at the ice-air interface may cause the solar flux within the ice to be sensitive to the solar zenith angle, irrespective of the absolute shortwave irradiance. To test this the model is rerun with a set of specific solar zenith angles covering the expected range experienced at the Frontier Mountain range (from $49^{\circ}$ to $89^{\circ}$ at $10^{\circ}$ intervals) with the mean bubble parameter set in all cases. In Fig. S1a the impact of this change can be seen in the reduction of solar flux with increasing SZA, particularly at the highest angle of incidence. Also shown are the results for

15 a diffuse sky which lies close to the $69^{\circ}$ result. The reduction in the downwelling fluxes is principally due to the high incidence angle rather than an increased scattering path c.f. the vertical depth. This is expected as after $1 /(1-g)$ scattering events, the radiation field can be considered isotropic and retains no information of the initial beam direction. In Fig. S1b the solar zenith angle dependence of the spectral albedo is shown, exhibiting a much greater variation than for changing scattering coefficients (Fig. 5). The increase of solar-weighted albedo at greater solar zenith angles is not attributed to

20 increased scattering at $\lambda<1440 \mathrm{~nm}$, but to increases at longer wavelengths where the albedo arises purely due to the surface reflections.

However to more properly represent the solar attenuation in bubbled ice the incident atmospheric radiation field must be partitioned between direct and diffuse components. Using the libRadtran atmospheric radiative transfer model the direct beam contribution to the total shortwave irradiance is calculated for the high cloud, low cloud and clear sky cases (see

25 Appendix A). The fractional direct contribution at each SZA is then calculated from a weighted average of the three atmospheric cases. Due to longer atmospheric path lengths, the fractional contribution of the direct beam reduces with increases in SZA, and therefore counteracts the surface Fresnel effect. This is shown in Fig. S1c where, when partitioning between the direct and diffuse effect is included, the solar flux transmitted to a given depth is far less sensitive to the incident SZA, as is the variation in the spectral albedo (Fig. S1d). 


\section{S2 Impact of different surface environments}

While the analysis in S1 considers the influence of a varying solar elevation and the partitioning between the direct solar beam and the diffuse sky, the normalised incident solar spectrum itself was held constant. In this section we investigate the effect of different surface and atmospheric environments upon the incident solar spectrum and the propagation of the 5 integrated solar SW flux into bubbled ice. To that end we have selected locations at different latitudes and altitudes representing a range of environments across both polar regions. These are summarised in Table S1. In all cases we assume a minimum solar zenith angle (maximum elevation) as would occur at noon at the summer solstice. Total ozone columns are estimated from Diaz et al. (2004) for Antarctica and Salby (1996) for other sites. Albedos are taken from Riihela et al. (2013) for the Arctic Sea ice locations; for other sites they are taken as the June or December monthly mean albedo for a $2^{\circ} \times 2^{\circ}$

10 geographic area surrounding the location (estimated from MODIS data, available from neo.sci.gsfc.nasa.gov). Aerosol optical depths are from Tomasi et al. (2007) for southern hemisphere environments and von Hardenberg et al. (2012) for the northern hemisphere. For each site we used these atmospheric parameters as inputs for the libRadtran model (see Appendix A) to calculate a clear sky solar spectral irradiance and a cloudy sky spectral irradiance. To calculate the latter the cloud microphysical properties ( $\boldsymbol{r}_{\text {eff }}$ and liquid water content) are taken from Mioche et al. (2017) for the Arctic locations and

15 from Lachlan-Cope et al. (2016) for the Antarctic sites. For both cases we assume a stratus-type cloud, with its base located at an altitude of $1.4 \mathrm{~km}$ above ground level and a height of $1.3 \mathrm{~km}$. This is then scaled with location-specific monthly mean MODIS cloud optical depths.

This analysis produces 14 different incident solar spectra including the clear sky and low cloud cases from the original Frontier Mountain site. These spectra primarily differ in their wavelength-integrated total solar irradiance, but, as this is a 20 more easily and more widely measured quantity, we normalise the spectra accordingly to focus on the spectral aspect. In Fig. S2a these normalised spectra are grouped according to their clear sky or cloud classification where a clear differentiation can be seen between the two groupings: cloudy sky cases exhibit relatively lower values at IR wavelengths above $1450 \mathrm{~nm}$ due to absorption by water. Within the cloudy sky group, the spectral shift between shorter and longer wavelengths is dominated by the amount of water absorption at these wavelengths: those cases where the cloud optical depth is highest result in the

25 lowest irradiances in this wavelength region. Though there is less intra-group variation, the clear sky spectra also exhibit a progressive wavelength shift, but in this group it is dependent on the air mass factor (a function of both the solar zenith angle and altitude). Locations associated with smaller air mass factors (or slant paths) such as at Summit, Greenland, show the greatest contribution from IR wavelengths.

We expect this (cloud thickness or slant path dependent) spectral shift between IR and visible wavelengths to be exhibited

30 below the ice surface. As demonstrated in Fig. S2b, surface environments and atmospheric conditions that result in a relatively low irradiance at longer IR wavelengths, show higher mean fluxes at all depths below the surface per unit irradiance incident on the surface. This is due to a shift towards those visible wavelengths where absorption by ice is least. It should be stressed however the result explicitly assumes a unit incident irradiance. In absolute terms, the presence of a cloud 
layer will reduce the total irradiance incident on the ice surface, outweighing this effect. More generally it is notable that despite our efforts to sample atmospheric and surface environments widely, there is relatively little difference between the fractional mean irradiance as a function of depth. At a depth of $0.2 \mathrm{~m}$ variation in the incident spectrum causes up to $12 \%$ variation in the fractional mean irradiance; at a depth of $1 \mathrm{~m}$ this rises to $14 \%$, and at $2 \mathrm{~m}$ it has risen to $15 \%$.
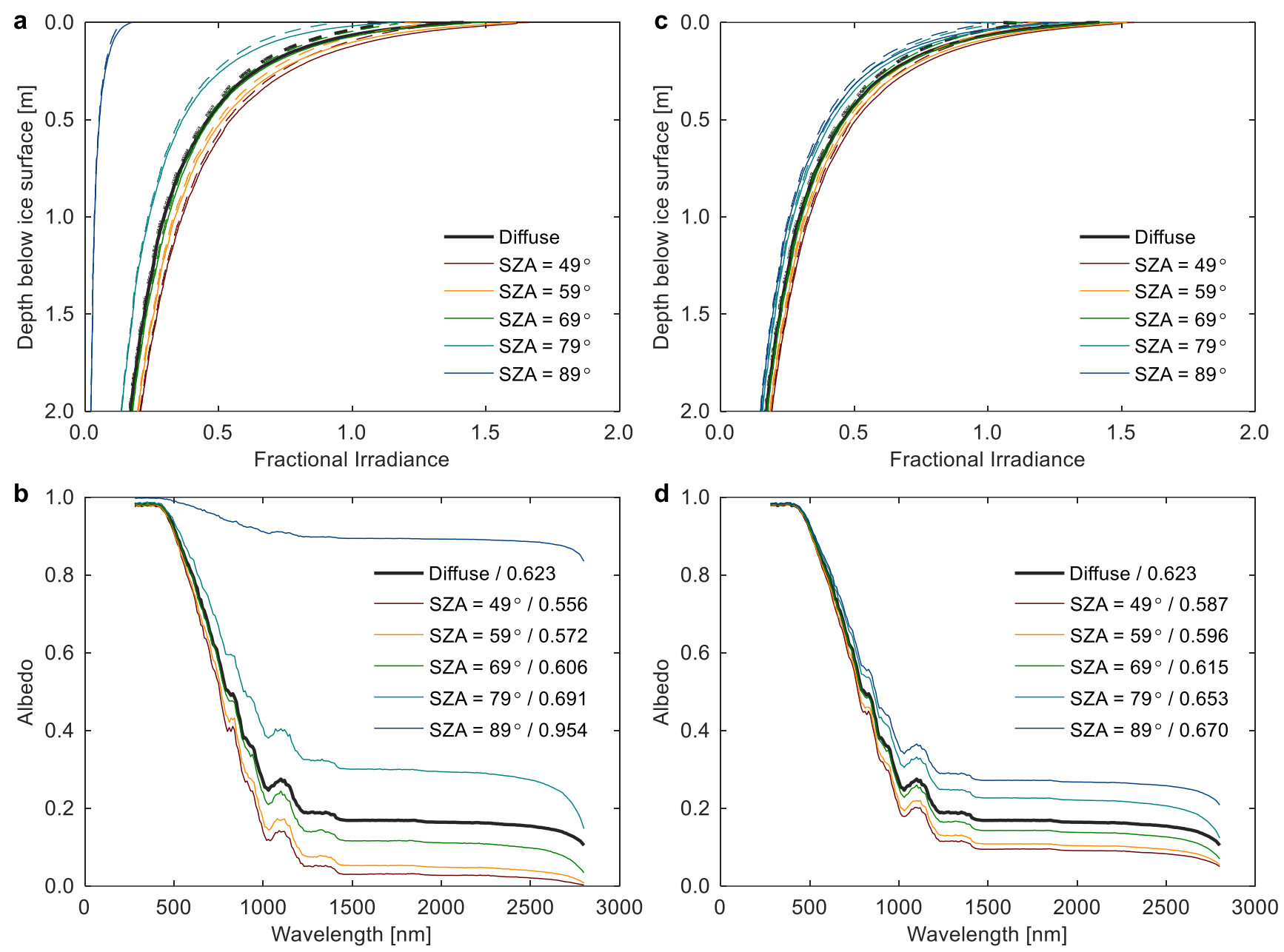

Figure S1. All calculations were carried out for the mean Dadic BIAs combined mCT and caliper parameter set $\left(r_{b u b}=229 \mu\right.$, $\left.N_{b u b}=1027 \mathrm{~cm}^{-3}\right)($ a) Fractional solar irradiance variation against ice depth for a diffuse sky and a range of incident solar zenith angles. Solid lines show the downwelling irradiance, whilst dotted lines show the upwelling irradiance. (b) Spectral albedo calculated for a diffuse sky and a range of incident solar zenith angles. Values in the legend are shortwave mean albedos weighted according to the incident solar spectrum. (c) Fractional solar irradiance variation against ice depth for a fully diffuse sky alongside results from separating the incident spectrum into direct and diffuse components for a range of incident solar zenith angles. Solid lines show the downwelling irradiance, whilst dotted lines show the upwelling irradiance. (d) Spectral albedo calculated for a fully diffuse sky alongside results from separating the incident spectrum into direct and diffuse components for a range of incident solar zenith angles. Values in the legend are shortwave mean albedos weighted according to the incident solar spectrum. 

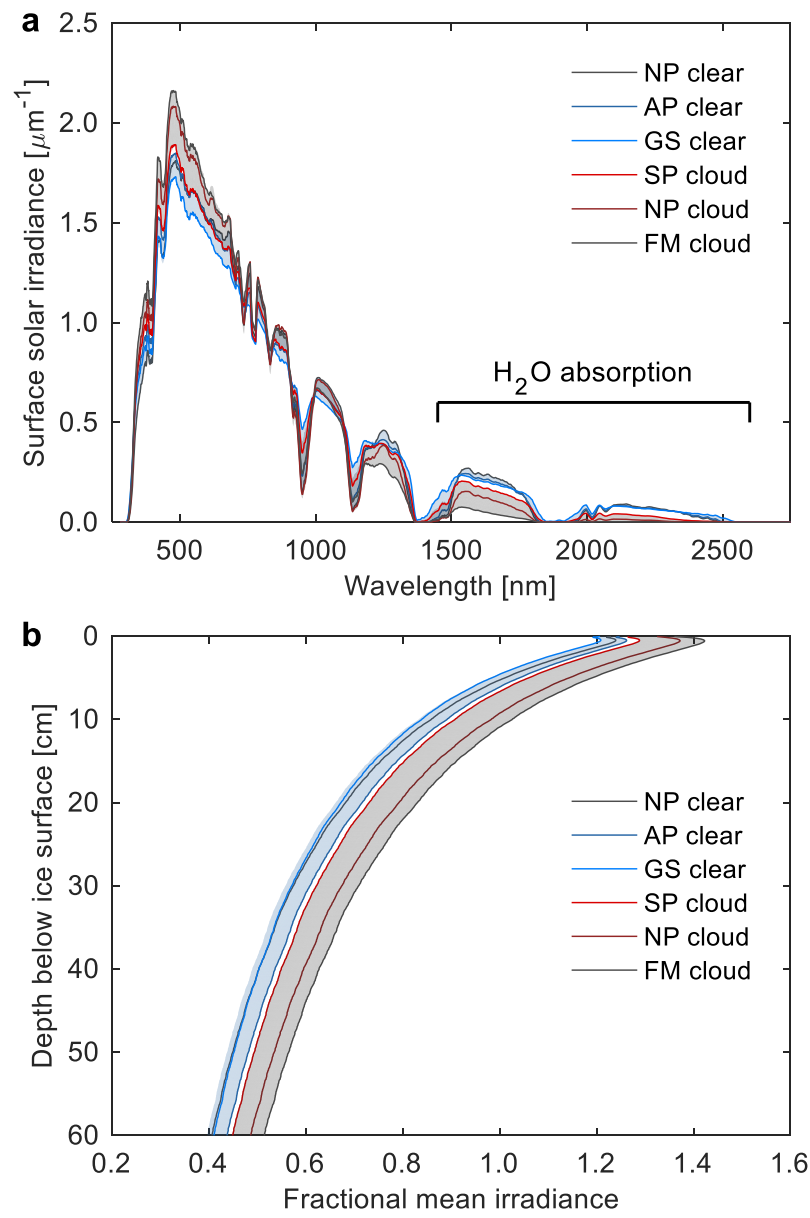

Figure S2. (a) Incident solar spectra calculated for a range of polar environments. Spectra are grouped according to clear sky or cloudy classification and a $20 \mathrm{~nm}$ running mean applied for clarity. Shaded areas show range of values within each classification 5 (grey for cloudy scenarios, blue for clear skies); individual spectra are selected according to their ranked cloud optical depth for cloudy skies (maximum, median and minimum) and ranked slant path for clear skies. (b) Fractional mean irradiance as a function of depth below ice-air interface for a range of polar environments. Again, lines are grouped according to clear sky or cloudy classification of incident solar spectrum. Shaded areas show range of values within each classification (grey for cloudy scenarios, blue for clear skies); selected individual traces are shown as in Fig. 8a, according to their ranked cloud optical depth or ranked 10 slant path. 
Table S1. Summary of atmospheric parameters used as input to libRadtran for location-specific clear sky and cloud solar spectral irradiance calculations. Data sources for total column ozone, albedo, aerosol optical depth (AOD) and cloud optical depth (COD) are as noted in the main text. Solar zenith angle (SZA) is the minimum SZA at the summer solstice, except for the Frontier Mountains where we retain the value used earlier.

\begin{tabular}{|c|c|c|c|c|c|c|}
\hline & $\begin{array}{l}\text { Ozone } \\
{[\mathrm{DU}]}\end{array}$ & $\begin{array}{c}\text { Altitude } \\
{[\mathrm{km}]}\end{array}$ & $\mathrm{SZA}\left[{ }^{\circ}\right]$ & Albedo & AOD & COD \\
\hline $\begin{array}{l}\text { South Pole (SP) } \\
{\left[90^{\circ} \mathrm{S}\right]}\end{array}$ & 280 & 2.83 & 66.5 & 0.81 & 0.015 & 9 \\
\hline $\begin{array}{l}\text { Frontier Mountains (FM) } \\
{\left[73^{\circ} \mathrm{S}, 161^{\circ} \mathrm{W}\right]}\end{array}$ & 300 & 2.04 & 66.4 & 0.62 & 0.028 & 50 \\
\hline $\begin{array}{l}\text { Antarctic Peninsula (AP) } \\
{\left[67^{\circ} \mathrm{S}, 68^{\circ} \mathrm{W}\right]}\end{array}$ & 300 & 0.016 & 43.5 & 0.74 & 0.029 & 22 \\
\hline $\begin{array}{l}\text { North Pole (NP) } \\
{\left[90^{\circ} \mathrm{N}\right]}\end{array}$ & 320 & 0.00 & 66.5 & 0.58 & 0.05 & 16 \\
\hline $\begin{array}{l}\operatorname{Arctic} \operatorname{Sea}(\mathrm{AS}) \\
{\left[82^{\circ} \mathrm{N}, 135^{\circ} \mathrm{W}\right]}\end{array}$ & 340 & 0.00 & 58.5 & 0.58 & 0.05 & 19 \\
\hline $\begin{array}{l}\text { Summit, Greenland (GS) } \\
{\left[72^{\circ} \mathrm{N}, 38^{\circ} \mathrm{W}\right]}\end{array}$ & 330 & 3.22 & 48.5 & 0.76 & 0.05 & 11 \\
\hline $\begin{array}{l}\text { Vatnajökull, Iceland (IC) } \\
{\left[64^{\circ} \mathrm{N}, 17^{\circ} \mathrm{W}\right]}\end{array}$ & 340 & 2.11 & 40.5 & 0.67 & 0.05 & 25 \\
\hline
\end{tabular}

